\title{
Controlling many-body states by the electric- field effect in a two-dimensional material
}

\author{
L. J. Li, E. C. T. O’Farrell, K. P. Loh, G. Eda, B. Özyilmaz \& A. H. Castro Neto*
}

\section{PAPER ABSTRACT}

To understand the complex physics of a system with strong electron-electron interactions, the ideal is to control and monitor its properties while tuning an external electric field applied to the system (the electric-field effect). Indeed, complete electric-field control of many-body states in strongly correlated electron systems is fundamental to the next generation of condensed matter research and devices. However, the material must be thin enough to avoid shielding of the electric field in the bulk material. Two-dimensional materials do not experience electrical screening, and their charge-carrier density can be controlled by gating. Octahedral titanium diselenide ( $\left(1 \mathrm{~T}-\mathrm{TiSe}_{2}\right.$ ) is a prototypical two-dimensional material that reveals a charge-density wave (CDW) and superconductivity in its phase diagram, presenting several similarities with other layered systems such as copper oxides, iron pnictides, and crystals of rare-earth elements and actinide atoms. By studying $1 \mathrm{~T}-\mathrm{TiSe}_{2}$ single crystals with thicknesses of 10 nanometres or less, encapsulated in two-dimensional layers of hexagonal boron nitride, we achieve unprecedented control over the CDW transition temperature (tuned from 170 kelvin to 40 kelvin), and over the superconductivity transition temperature (tuned from a quantum critical point at 0 kelvin up to 3 kelvin). Electrically driving TiSe ${ }_{2}$ over different ordered electronic phases allows us to study the details of the phase transitions between manybody states. Observations of periodic oscillations of magnetoresistance induced by the Little-Parks effect show that the appearance of superconductivity is directly correlated with the spatial texturing of the amplitude and phase of the superconductivity order parameter, corresponding to a two-dimensional matrix of superconductivity. We infer that this superconductivity matrix is supported by a matrix of incommensurate CDW states embedded in the commensurate CDW states. Our results show that spatially modulated electronic states are fundamental to the appearance of two-dimensional superconductivity.

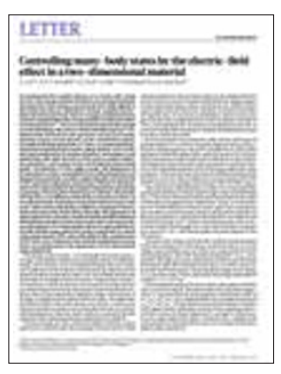
DETAILS

Citation: Nature 529, 185-189 (2016)

doi: 10.1038/nature 16175

- RECEIVED 12 April 2015

- ACCEPTED 19 October 2015

- PUBLISHED ONLINE 23 December 2015

\section{SUMMARY}

Controlling electrons in two dimensions. It is now possible to manipulate the behaviour of electrons in 2D materials nanometres thick by varying an external electric field, opening new routes to controlling electronic materials and devices.

\section{The challenge}

Scientists and engineers have dreamed of controlling the number of electrons in materials by simply applying external electric fields to devices $^{1-3}$. Historically, this control has been achieved by chemical doping, the synthesis of new materials with different chemical composition. Although powerful, chemical doping irreversibly changes the material and creates disorder. Sometimes hundreds of different samples have to be created to obtain the desired effect. In theory, many different electronic states could be observed in a single sample if the electron behaviour could be controlled externally. By simply modifying the number of electrons, or carrier concentration, the material switches between different electronic states, including metals, semiconductors, electronic crystals (the so-called charge density waves, CDW), magnets (the socalled spin density waves, SDW), and superconductors. Such control has not been possible in classic 3D materials because the electrons move in three dimensions and screen the external electric field.

\section{The solution}

The electrons in atomically thin 2D materials cannot shield external electric fields because they are confined to move in only two directions. Hence, 3D electric fields can change the charge concentration of 2D materials without any chemical manipulation. By varying the device voltage and its temperature, the entire phase diagram of a material can be explored. With one sample it is now possible to study what previously required hundreds of individually synthesized samples.

The key to our approach is the quality of both the material and the device. We chose a single $2 \mathrm{D}$ crystal of titanium diselenide, $\mathrm{TiSe}_{2}$, less than 10 nanometres thick, and protected it from the environment by sandwiching it between another 2D material, boron nitride, which is an 
inert insulator. These crystals were mounted on a device with bottom and top gate (an ionic gel) - see Figure. The device was also loaded into a cryostat that can alter the temperature from $-270^{\circ} \mathrm{C}$ to $-27^{\circ} \mathrm{C}$.

We observed and measured with great accuracy the phase transitions between metal, semiconducting CDW, and superconductor by varying the electric field and temperature. We varied the electron numbers between $10^{14} \mathrm{~cm}^{-2}$ and $10^{15} \mathrm{~cm}^{-2}$, and observed the CDW transition temperature decrease from $170 \mathrm{~K}$ to $40 \mathrm{~K}$ across this range. We also observed the superconductivity transition increase from $0 \mathrm{~K}$ to $3 \mathrm{~K}$ as the electron density rises. The point at which superconductivity first appears at $0 \mathrm{~K}$ is called a quantum critical point and is dominated by pure quantum fluctuations, not thermal ones ${ }^{4}$. Surprisingly, we also observed unusual oscillations of the electrical resistance in the superconducting phase when a magnetic field is applied ${ }^{5}$. Those oscillations imply that the CDW and 2D superconductivity states are spatially correlated ${ }^{6}$. This effect was never observed in the $3 \mathrm{D}$ version of the same material.

\section{Future directions}

Many other materials, including high-temperature and heavy-fermion superconductors, have essentially the same phase diagram as $\mathrm{TiSe}_{2}$. Our results point to new investigations of all these materials, whose behaviour is much debated, within a well controlled device. Nobel laureate Herbert Kroemer said that "the interface is the device". As materials become atomically thin, $2 \mathrm{D}$ crystals become pure interfaces. We have shown that our crystal can be much more than a single device; it can be a tool for the investigation of new electronic states. If we can harness these electronic states to develop new functionalities for exploiting magnetism and superconductivity, for instance, the possibilities are endless.

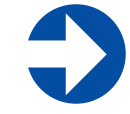

The full version of the original article is on Nature's website at go.nature.com/28xfkxf

\section{GRAPHICAL ABSTRACT}

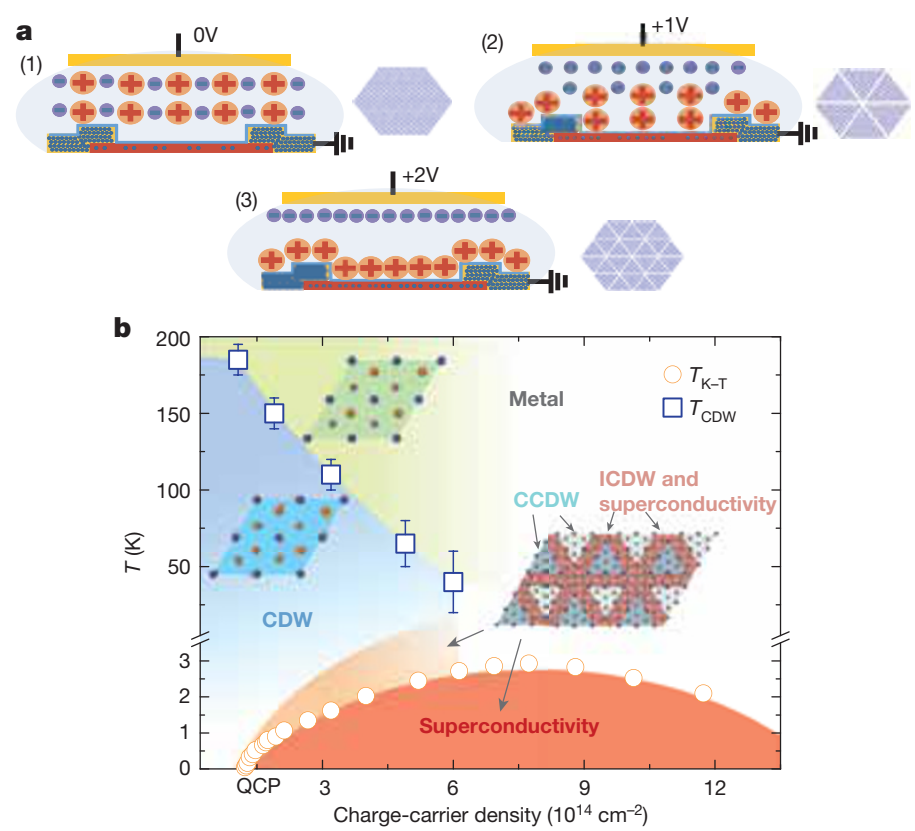

Manipulating electrons in a nanoscale material. a, Device operation. When the electric field is applied to the ion gel it polarizes strongly, creating a large electric field in the $2 \mathrm{D}$ material. As the polarization in the ion gel increases, increasing the charge density, the CDW domain size in the $2 \mathrm{D}$ material decreases. We observe oscillations in the electrical conductivity as a function of applied magnetic field that indicate the formation of domains via the so-called Little-Parks effect ${ }^{5}$. $\mathbf{b}$, Phase diagram obtained by applying the electric field. Vertical axis is the temperature (controlled in the cryostat) and horizontal axis is charge density (which is controlled by the applied electric field). We show the three different phases, namely, metal, semiconducting CDW, and superconductor. The insets illustrate the position of the atoms in the lattice in each phase. In the superconducting phase we show that the CDW breaks down into domains (as shown in (a)) and superconductivity develops at the boundaries of the domains.

\section{FURTHER READING}

1. Ueno, K. et al. Electric-field-induced superconductivity in an insulator. Nature Mater. 7, 855-858 (2008).

This study shows that electric fields can be applied to a 3D insulator to make it into a low-temperature superconductor at the topmost surface.

2. Yu, Y. et al. Gate-tunable phase transitions in thin flakes of $1 \mathrm{~T}-\mathrm{TaS}_{2}$. Nature Nanotechnol. 10, 270-276 (2015).

This paper discusses a material similar to $\mathrm{TiSe}_{2}$, namely $\mathrm{TaS}_{2}$, in which superconductivity is induced by $\mathrm{Li}$ intercalation in thin samples.

3. Bollinger, A. T. et al. Superconductor-insulator transition in $\mathrm{La}_{2-x} \mathrm{Sr}_{x} \mathrm{CuO}_{4}$ at the pair quantum resistance. Nature 472, 458-460 (2011). A thin film of the non-stoichimetric hightemperature superconductor LSCO is grown by molecular beam epitaxy and its transport properties are measured.

4. Sachdev, S. Ouantum Phase Transitions (Cambridge Univ. Press, 1999).

This book gives a comprehensive description of quantum phase transitions and quantum critical points in many different situations.

5. Tinkham, M. Introduction to Superconductivity (McGraw-Hill, 1975).

This is a classic book on superconductivity and has a description of the LittleParks effect, which is the basis for the interpretation of our magneto-resistance data.

6. Joe, Y. I. et al. Emergence of charge density wave domain walls above the superconducting dome in $1 \mathrm{~T}-\mathrm{TiSe}_{2}$. Nature Phys. 10, 421-425 (2014).

This study shows the formation of CDW domains in 3D $\mathrm{TiSe}_{2}$ close to the superconducting phase.

\section{RELATED CONTENT}

\author{
Inhomogeneity of charge-density-wave \\ order and quenched disorder in a high- $\mathrm{T}_{c}$ \\ superconductor \\ G. Campi et al. \\ go.nature.com/1poqg9t
}

Theory of intertwined orders in high temperature superconductors

E. Fradkin et al.

go.nature.com/25tceov

Electric field effect in atomically thin carbon films

K. S. Novoselov et al.

go.nature.com/1sc9g6b 